\title{
Special issue on computational heat transfer and fluid dynamics
}

\author{
Arvind Pattamatta $^{1} \cdot$ Balaji Srinivasan $^{1} \cdot$ Sateesh Gedupudi $^{1}$
}

Published online: 13 November 2018

(C) Indian Institute of Technology Madras 2018

The Asian Symposium on Computational Heat Transfer (ASCHT) conference has become a unique platform for discussion of ideas and sharing of knowledge in the field of computational methods in heat transfer and fluid flow. Over the years, it has brought together many experts of the field from academia and the industry. It has thus proved to be immensely fruitful for budding researchers in the field. Accordingly, the sixth edition of ASCHT (2017) was hosted at the Department of Mechanical Engineering, Indian Institute of Technology Madras, Chennai (India). The response to the conference announcement was very good. A total number of 193 full manuscripts were received. All submissions were reviewed by at least two experts in the subject domain. In the final form, the proceedings include 155 papers that have been found suitable for presentation at the symposium. These cover a variety of topics in the traditional as well as futuristic areas of computational heat transfer and fluid flow.

The two special issues of International Journal of Advances in Engineering Sciences and Applied Mathematics include thirteen carefully chosen peer reviewed papers focused on various topics of current interest in Computational heat transfer and fluid dynamics.

The seven papers presented in the current issue (Special Issue II) deal with a wide range of computational aspects of heat transfer and fluid dynamics. The first article presents the condition number analysis of pressure and velocity matrices arising from an unstructured finite volume formulation of Navier-Stokes equations. The condition number-based algorithm resulted in an overall reduction in the simulation time. In

Sateesh Gedupudi

sateeshg@iitm.ac.in

1 Chennai, India the next article, a voxelized computational fluid dynamics model based on dynamic contrast enhanced-magnetic resonance imaging (DCE-MRI) has been employed to simulate the transport of chemotherapeutic drug (doxorubicin) in human brain tumors. Transient drug concentration in tumor tissue is predicted using the steady state interstitial fluid pressure and velocity values. The subsequent article statistically discusses the influence of the dynamical effects of rough wall turbulence on skin friction coefficient considering the randomly distributed semispheres. Direct numerical simulations are performed using the multiple-relaxation-time lattice Boltzmann method. This is followed by an article that presents a layout of two dimensional thermal network model for Thermal Interface Material subjected to a high frequency pulsed heat source. The approach characterizes the importance of two dimensional electrical resistance capacitance network modelling to capture thermal resistances and associated delays in both dimensions. The next article deals with the transient hydrodynamic and heat transfer behavior of a cross flow over a heated circular cylinder under the influence of thermal buoyancy. Numerical simulations have been performed using Ghost-Fluid Thermal Lattice Boltzmann method. The subsequent article clarifies the dissimilarity between heat and momentum transport for a turbulent stripe. Direct numerical simulations using a spectral method of plane Couette flow are performed. The last article presents a Multi-Time-Scale Turbulent Heat Transfer Model for Predictions of Various Turbulent Heat Transfer Phenomena. Improvement of the prediction performance of the proposed model is demonstrated for complex fields of turbulent heat transfer problem.

The guest editors take this opportunity to thank the authors for communicating the revised papers to the special issues, the referees for their timely and critical reviews, and the Springer publishing team for their cooperation. 